Kragujevac Journal of Mathematics

Volume 44(3) (2020), Pages 335-351.

\title{
MORE ABOUT PETROVIĆ'S INEQUALITY ON COORDINATES VIA $m$-CONVEX FUNCTIONS AND RELATED RESULTS
}

\author{
ATIQ UR REHMAN ${ }^{1}$, GHULAM FARID $^{1}$, AND WASIM IQBAL $^{2}$
}

\begin{abstract}
In this paper the authors extend Petrović's inequality for coordinated $m$-convex functions in the plane and also find Lagrange type and Cauchy type mean value theorems for Petrović's inequality for $m$-convex functions and coordinated $m$-convex functions. The authors consider functional due to Petrović's inequality in plane and discuss its properties for certain class of coordinated log- $m$-convex functions.
\end{abstract}

\section{INTRODUCTION}

A function $f:[a, b] \rightarrow \mathbb{R}$ is said to be convex if

$$
f(t x+(1-t) y) \leq t f(x)+(1-t) f(y)
$$

holds, for all $x, y \in[a, b]$ and $t \in[0,1]$.

In [6], Dragomir gave the definition of convex functions on coordinates as follows.

Definition 1.1. Let $\Delta=[a, b] \times[c, d] \subseteq \mathbb{R}^{2}$ and $f: \Delta \rightarrow \mathbb{R}$ be a mapping. Define partial mappings

$$
f_{y}:[a, b] \rightarrow \mathbb{R} \text { by } f_{y}(u)=f(u, y)
$$

and

$$
f_{x}:[c, d] \rightarrow \mathbb{R} \text { by } f_{x}(v)=f(x, v) .
$$

Then $f$ is said to be convex on coordinates (or coordinated convex) in $\Delta$ if $f_{y}$ and $f_{x}$ are convex on $[a, b]$ and $[c, d]$ respectively for all $y \in[c, d]$ and $x \in[a, b]$. A mapping $f$ is said to be strictly convex on coordinates (or strictly coordinated convex) in $\Delta$

Key words and phrases. Petrović's inequality, mean value theorem, log-convexity, $m$-convex functions on coordinates.

2010 Mathematics Subject Classification. 26A51, 26D15, 35B05.

DOI 10.46793/KgJMat2003.335R

Received: January 24, 2018.

Accepted: April 30, 2018. 
if $f_{y}$ and $f_{x}$ are strictly convex on $[a, b]$ and $[c, d]$, respectively, for all $y \in[c, d]$ and $x \in[a, b]$.

In [22], G. Toader gave the definition of $m$-convexity as follows.

Definition 1.2. The function $f:[0, b] \rightarrow \mathbb{R}, b>0$, is said to be $m$-convex, where $m \in[0,1]$, if we have

$$
f(t x+m(1-t) y) \leqslant t f(x)+m(1-t) f(y),
$$

for all $x, y \in[0, b]$ and $t \in[0,1]$.

Remark 1.1. One can note that the notion of $m$-convexity reduces to convexity if we take $m=1$. We get starshaped functions from $m$-convex functions if we take $m=0$.

Definition 1.3. A function $f:[a, b] \rightarrow \mathbb{R}_{+}$is called log-convex if

$$
f(t x+(1-t) y) \leqslant f^{t}(x)+f^{(1-t)}(y)
$$

holds, for all $x, y \in[0, b]$ and $t \in[0,1]$.

Log-convex functions have excellent closure properties. The sum and product of two log-convex functions is convex. If $f$ is convex function and $g$ is log-convex function then the functional composition $g \circ f$ is also log-convex.

In [1], Almori and Darus gave the definition of log-convex on coordinates as follows.

Definition 1.4. Let $\Delta=[a, b] \times[c, d]$ and let a function $f: \Delta \rightarrow \mathbb{R}_{+}$is called log-convex on coordinates in $\Delta$ if partial mappings defined in (1.1) and (1.2) are log-convex on $[a, b]$ and $[c, d]$, respectively, for all $y \in[c, d]$ and $x \in[a, b]$.

In [8], Farid et al. gave the definition of coordinated $m$-convex functions as follows.

Definition 1.5. Let $\Delta=[0, b] \times[0, d] \subset[0, \infty)^{2}$, then a function $f: \Delta \rightarrow \mathbb{R}$ will be called $m$-convex on coordinates if the partial mappings

$$
f_{y}:[0, b] \rightarrow \mathbb{R} \text { defined by } f_{y}(u)=f(u, y)
$$

and

$$
f_{x}:[0, d] \rightarrow \mathbb{R} \text { defined by } f_{x}(v)=f(x, v)
$$

are $m$-convex on $[0, b]$ and $[0, d]$, respectively, for all $y \in[0, d]$ and $x \in[0, b]$.

In [17] (see also [15, p. 154]), M. Petrović proved the following result, which is known as Petrović's inequality in the literature.

Theorem 1.1. Suppose that $\left(x_{1}, \ldots, x_{n}\right)$ and $\left(p_{1}, \ldots, p_{n}\right)$ be two non-negative $n$-tuples such that $\sum_{k=1}^{n} p_{k} x_{k} \geq x_{i}$ for $i=1, \ldots, n$ and $\sum_{k=1}^{n} p_{k} x_{k} \in[0, a]$. If $f$ is a convex function on $[0, a)$, then the inequality

$$
\sum_{k=1}^{n} p_{k} f\left(x_{k}\right) \leq f\left(\sum_{k=1}^{n} p_{k} x_{k}\right)+\left(\sum_{k=1}^{n} p_{k}-1\right) f(0)
$$

is valid. 
Remark 1.2. Take $p_{k}=1, k=1, \ldots, n$ the above inequality becomes

$$
\sum_{k=1}^{n} f\left(x_{k}\right) \leq f\left(\sum_{k=1}^{n} x_{k}\right)+(n-1) f(0) .
$$

In [2], M. Bakula et al. gave the Petrović's inequality for $m$-convex function which is stated in the following theorem.

Theorem 1.2. Let $\left(x_{1}, \ldots, x_{n}\right)$ be non-negative $n$-tuples and $\left(p_{1}, \ldots, p_{n}\right)$ be positive $n$-tuples such that

$$
P_{n}:=\sum_{k=1}^{n} p_{k}, \quad 0 \neq \tilde{x}_{n}=\sum_{k=1}^{n} p_{k} x_{k} \geq x_{j} \text { for each } j=1, \ldots, n .
$$

If $f:[0, \infty) \rightarrow \mathbb{R}$ be an $m$-convex function on $[0, \infty)$ with $m \in(0,1]$, then

$$
\sum_{k=1}^{n} p_{k} f\left(x_{k}\right) \leqslant \min \left\{m f\left(\frac{\tilde{x}_{n}}{m}\right)+\left(P_{n}-1\right) f(0), f\left(\tilde{x}_{n}\right)+m\left(P_{n}-1\right) f(0)\right\} .
$$

Remark 1.3. If we take $m=1$ in Theorem 1.2, we get famous Petrović's inequality stated in Theorem 1.1.

In [19], Rehman et al. gave the Petrović's inequality for coordinated convex functions, which is stated in the following theorem.

Theorem 1.3. Let $\left(x_{1}, \ldots, x_{n}\right) \in[0, a)^{n},\left(y_{1}, \ldots, y_{n}\right) \in[0, b)^{n}$ and $\left(p_{1}, \ldots, p_{n}\right)$, $\left(q_{1}, \ldots, q_{n}\right)$ be positive $n$-tuples such that $\sum_{k=1}^{n} p_{k} x_{k} \in[0, a), \sum_{j=1}^{n} q_{j} y_{j} \in[0, b)$, $\sum_{k=1}^{n} p_{k} \geq 1$,

$$
P_{n}:=\sum_{k=1}^{n} p_{k}, \quad 0 \neq \tilde{x}_{n}=\sum_{k=1}^{n} p_{k} x_{k} \geq x_{i} \text { for each } i=1, \ldots, n,
$$

and

$$
Q_{n}:=\sum_{j=1}^{n} q_{j}, \quad 0 \neq \tilde{y}_{n}=\sum_{j=1}^{n} q_{j} y_{j} \geq y_{i} \text { for each } i=1, \ldots, n .
$$

If $f: \Delta \rightarrow \mathbb{R}$ be a coordinated convex, then

$$
\begin{aligned}
\sum_{k=1}^{n} \sum_{j=1}^{n} p_{k} q_{j} f\left(x_{k}, y_{j}\right) \leq & f\left(\tilde{x}_{n}, \tilde{y}_{n}\right)+\left(Q_{n}-1\right) f\left(\tilde{x}_{n}, 0\right) \\
& +\left(P_{n}-1\right)\left(f\left(0, \tilde{y}_{n}\right)+\left(Q_{n}-1\right) f(0,0)\right) .
\end{aligned}
$$

By considering non-negative difference of (1.5), the authors in [19] defined the following functional

$$
\begin{aligned}
\Upsilon(f)= & f\left(\tilde{x}_{n}, \tilde{y}_{n}\right)+\left(Q_{n}-1\right) f\left(\tilde{x}_{n}, 0\right)+\left(P_{n}-1\right)\left[f\left(0, \tilde{y}_{n}\right)+\left(Q_{n}-1\right) f(0,0)\right] \\
& -\sum_{k=1}^{n} \sum_{j=1}^{n} p_{k} q_{j} f\left(x_{k}, y_{j}\right) .
\end{aligned}
$$


By considering non-negative difference of (1.3), the authors in [4] defined the following functional

$$
\mathcal{P}(f)=f\left(\sum_{k=1}^{n} p_{k} x_{k}\right)-\left(\sum_{k=1}^{n} p_{k} f\left(x_{k}\right)\right)+\left(\sum_{k=1}^{n} p_{k}-1\right) f(0) .
$$

One of the generalizations of convex functions is $m$-convex functions and it is considered in literature by many researchers and mathematicians, for example, see $[7,10-12,24]$ and references there in. In [17] (also see [15, p. 154]), M. Petrović gave the inequality for convex functions known as Petrović's inequality. Many authors worked on this inequality by giving results related to it, for example see $[13,15,17]$ and it has been generalized for $m$-convex functions by M. Bakula et al. in [2]. In [19], Petrović's inequality was generalized on coordinate by using the definition of convex functions on coordinates given by Dragomir in [6].

In this paper the authors extend Petrović's inequality for coordinated $m$-convex functions in the plane and also find Lagrange type and Cauchy type mean value theorems for Petrović's inequality for $m$-convex functions and coordinated $m$-convex functions. The authors consider functional due to Petrović's inequality in plane and discuss its properties for certain class of coordinated log- $m$-convex functions.

\section{Main Result}

The following theorem consist the result for Petrović's inequality on coordinated $m$-convex functions.

Theorem 2.1. Let $\left(x_{1}, \ldots, x_{n}\right),\left(y_{1}, \ldots, y_{n}\right)$ be non-negative $n$-tuples and $\left(p_{1}, \ldots, p_{n}\right)$, $\left(q_{1}, \ldots, q_{n}\right)$ be positive $n$-tuples such that $\sum_{k=1}^{n} p_{k} \geq 1$,

$$
P_{n}:=\sum_{k=1}^{n} p_{k}, \quad 0 \neq \tilde{x}_{n}=\sum_{k=1}^{n} p_{k} x_{k} \geq x_{i} \text { for each } i=1, \ldots, n
$$

and

$$
Q_{n}:=\sum_{j=1}^{n} q_{j}, \quad 0 \neq \tilde{y}_{n}=\sum_{j=1}^{n} q_{j} y_{j} \geq y_{i} \text { for each } i=1, \ldots, n .
$$

If $f:[0, \infty)^{2} \rightarrow \mathbb{R}$ be an $m$-convex function on coordinates with $m \in(0,1]$, then

$$
\begin{aligned}
\sum_{k=1}^{n} \sum_{j=1}^{n} p_{k} q_{j} f\left(x_{k}, y_{j}\right) \leq & \min \left\{m \min \left\{G_{m, 1}\left(\tilde{x}_{n} / m\right), G_{1, m}\left(\tilde{x}_{n} / m\right)\right\}+\left(P_{n}-1\right)\right. \\
& \times \min \left\{G_{m, 1}(0), G_{1, m}(0)\right\}, \min \left\{G_{m, 1}\left(\tilde{x}_{n}\right), G_{1, m}\left(\tilde{x}_{n}\right)\right\} \\
& \left.+m\left(P_{n}-1\right) \min \left\{G_{m, 1}(0), G_{1, m}(0)\right\}\right\}
\end{aligned}
$$

where

$$
G_{m, \widetilde{m}}(t)=m f\left(t, \frac{\tilde{y}_{n}}{m}\right)+\widetilde{m}\left(Q_{n}-1\right) f(t, 0) .
$$


Proof. Let $f_{x}:[0, \infty) \rightarrow \mathbb{R}$ and $f_{y}:[0, \infty) \rightarrow \mathbb{R}$ be mappings such that $f_{x}(v)=f(x, v)$ and $f_{y}(u)=f(u, y)$. Since $f$ is coordinated $m$-convex on $[0, \infty)^{2}$, therefore $f_{y}$ is $m$ convex on $[0, \infty)$, so by Theorem 1.2 , one has

$$
\sum_{k=1}^{n} p_{k} f_{y}\left(x_{k}\right) \leq \min \left\{m f_{y}\left(\tilde{x}_{n} / m\right)+\left(P_{n}-1\right) f_{y}(0), f_{y}\left(\tilde{x}_{n}\right)+m\left(P_{n}-1\right) f_{y}(0)\right\} .
$$

This is equivalent to

$$
\begin{gathered}
\sum_{k=1}^{n} p_{k} f\left(x_{k}, y\right) \leq \min \left\{m f\left(\tilde{x}_{n} / m, y\right)+\left(P_{n}-1\right) f(0, y),\right. \\
\left.f\left(\tilde{x}_{n}, y\right)+m\left(P_{n}-1\right) f(0, y)\right\} .
\end{gathered}
$$

By setting $y=y_{j}$, we have

$$
\begin{gathered}
\sum_{k=1}^{n} p_{k} f\left(x_{k}, y_{j}\right) \leq \min \left\{m f\left(\tilde{x}_{n} / m, y_{j}\right)+\left(P_{n}-1\right) f\left(0, y_{j}\right),\right. \\
\left.f\left(\tilde{x}_{n}, y_{j}\right)+m\left(P_{n}-1\right) f\left(0, y_{j}\right)\right\}
\end{gathered}
$$

this gives

$$
\begin{aligned}
\sum_{k=1}^{n} \sum_{j=1}^{n} p_{k} q_{j} f\left(x_{k}, y_{j}\right) \leq & \min \left\{m \sum_{j=1}^{n} q_{j} f\left(\tilde{x}_{n} / m, y_{j}\right)+\left(P_{n}-1\right) \sum_{j=1}^{n} q_{j} f\left(0, y_{j}\right),\right. \\
& \left.\sum_{j=1}^{n} q_{j} f\left(\tilde{x}_{n}, y_{j}\right)+m\left(P_{n}-1\right) \sum_{j=1}^{n} q_{j} f\left(0, y_{j}\right)\right\} .
\end{aligned}
$$

Now again by Theorem 1.2, one has

$$
\begin{aligned}
\sum_{j=1}^{n} q_{j} f\left(\tilde{x}_{n} / m, y_{j}\right) \leq & \min \left\{m f\left(\tilde{x}_{n} / m, \tilde{y}_{n} / m\right)+\left(Q_{n}-1\right) f\left(\tilde{x}_{n} / m, 0\right),\right. \\
& \left.f\left(\tilde{x}_{n} / m, \tilde{y}_{n}\right)+m\left(Q_{n}-1\right) f\left(\tilde{x}_{n} / m, 0\right)\right\} \\
\sum_{j=1}^{n} q_{j} f\left(0, y_{j}\right) \leq & \min \left\{m f\left(0, \tilde{y}_{n} / m\right)+\left(Q_{n}-1\right) f(0,0),\right. \\
& \left.f\left(0, \tilde{y}_{n}\right)+m\left(Q_{n}-1\right) f(0,0)\right\}
\end{aligned}
$$

and

$$
\begin{gathered}
\sum_{j=1}^{n} q_{j} f\left(\tilde{x}_{n}, y_{j}\right) \leq \min \left\{m f\left(\tilde{x}_{n}, \tilde{y}_{n} / m\right)+\left(Q_{n}-1\right) f\left(\tilde{x}_{n}, 0\right),\right. \\
\left.f\left(\tilde{x}_{n}, \tilde{y}_{n}\right)+m\left(Q_{n}-1\right) f\left(\tilde{x}_{n}, 0\right)\right\} .
\end{gathered}
$$

Putting these values in inequality (2.3), and using the notation in (2.2), one has the required result.

Remark 2.1. If we take $m=1$ in Theorem 2.1, we get Theorem 1.3.

In the following corollary, we gave new Petrović's type inequality for $m$-convex functions. 
Corollary 2.1. Let $\left(x_{1}, \ldots, x_{n}\right),\left(y_{1}, \ldots, y_{n}\right)$ be non-negative $n$-tuples and $\left(p_{1}, \ldots, p_{n}\right)$, $\left(q_{1}, \ldots, q_{n}\right)$ be positive $n$-tuples such that $\sum_{k=1}^{n} p_{k} \geq 1$ and

$$
P_{n}:=\sum_{k=1}^{n} p_{k}, \quad 0 \neq \tilde{x}_{n}=\sum_{k=1}^{n} p_{k} x_{k} \geq x_{i} \text { for each } i=1, \ldots, n .
$$

If $f:[0, \infty)^{2} \rightarrow \mathbb{R}$ be an $m$-convex function on coordinates with $m \in(0,1]$, then one has

$$
\text { (2.4) } \begin{aligned}
\sum_{k=1}^{n} n p_{k} f\left(x_{k}\right) \leq & \min \left\{m \min \left\{(m+n-1) f\left(\tilde{x}_{n} / m\right),(m n-m+1) f\left(\tilde{x}_{n} / m\right)\right\}\right. \\
& +\left(P_{n}-1\right) \min \{(m+n-1) f(0),(m n-m+1) f(0)\} \\
& \min \left\{(m+n-1) f\left(\tilde{x}_{n}\right),(m n-m+1) f\left(\tilde{x}_{n}\right)\right\} \\
& \left.+m\left(P_{n}-1\right) \min \{(m+n-1),(m n-m+1) f(0)\}\right\} .
\end{aligned}
$$

Proof. If we put $y_{j}=0$ and $q_{j}=1, j=1, \ldots, n$ with $f(x, 0) \mapsto f(x)$ in inequality (2.1), we get the required result.

Remark 2.2. If we take $m=1$ in inequality (2.4), we get the inequality (1.3).

Let $f:[0, b] \rightarrow \mathbb{R}$ be a function. Then we define

$$
P_{a, m, f}(x):=\frac{f(x)-m f(a)}{x-m a},
$$

for all $x \in[0, b] \backslash\{m a\}$, for fixed $a \in[0, b]$. Also define

$$
r_{m}\left(x_{1}, x_{2}, x_{3} ; f\right):=\frac{P_{x_{1}, m}\left(x_{3}\right)-P_{x_{1}, m}\left(x_{2}\right)}{x_{3}-x_{2}},
$$

where $x_{1}, x_{2}, x_{3} \in[0, b],\left(x_{2}-m x_{1}\right)\left(x_{3}-m x_{1}\right)>0, x_{2} \neq x_{3}$.

In [11] (see also [7, p. 294]), V. G. Mihesan considered the functions defined in (2.5), (2.6) and proved the following result.

Remark 2.3. If we take $m=1$ in (2.5) and (2.6), we get divided differences of first and second order respectively.

By considering non-negative difference of (1.4), we defined following functional

$$
\mathcal{P}_{m}(f)=\min \left\{m f\left(\frac{\tilde{x}_{n}}{m}\right)+\left(P_{n}-1\right) f(0), f\left(\tilde{x}_{n}\right)+m\left(P_{n}-1\right) f(0)\right\}-\sum_{k=1}^{n} p_{k} f\left(x_{k}\right) .
$$

Also by considering non-negative difference of (2.1), we defined following functional

$$
\begin{aligned}
\Upsilon_{m}(f)= & \min \left\{m \min \left\{G_{m, 1}\left(\tilde{x_{n}} / m\right), G_{1, m}\left(\tilde{x_{n}} / m\right)\right\}\right. \\
& +\left(P_{n}-1\right) \min \left\{G_{m, 1}(0), G_{1, m}(0)\right\}, \min \left\{G_{m, 1}\left(\tilde{x_{n}}\right), G_{1, m}\left(\tilde{x_{n}}\right)\right\} \\
& \left.+m\left(P_{n}-1\right) \min \left\{G_{m, 1}(0), G_{1, m}(0)\right\}\right\}-\sum_{k=1}^{n} \sum_{j=1}^{n} p_{k} q_{j} f\left(x_{k}, y_{j}\right) .
\end{aligned}
$$

If we take $m=1$ in the above $(2.8)$, we get $\Upsilon_{1}(f)=\Upsilon(f)$. 
Remark 2.4. Under the suppositions of Theorem 2.1, if $f$ is coordinated $m$-convex in $\Delta^{2}$, then $\Upsilon_{m}(f) \geq 0$.

Here we state an important lemma that is very helpful in proving mean value theorems related to the non-negative functional of Petrović's inequality for $m$-convex functions.

Lemma 2.1. Let $f:[0, b] \rightarrow \mathbb{R}$ be a function such that

$$
m_{1} \leqslant \frac{(x-m a) f^{\prime}(x)-f(x)+m f(a)}{x^{2}-2 m a x+m a^{2}} \leqslant M_{1},
$$

for all $x \in[0, b] \backslash\{m a\}, a \in(0, b)$ and $m \in(0,1)$.

Consider the functions $\psi_{1}, \psi_{2}:[0, b] \rightarrow \mathbb{R}$ defined as

$$
\psi_{1}(x)=M_{1} x^{2}-f(x)
$$

and

$$
\psi_{2}(x)=f(x)-m_{1} x^{2}
$$

then $\psi_{1}$ and $\psi_{2}$ are $m$-convex in $[0, b]$.

Proof. Suppose

So we have

$$
\begin{aligned}
P_{a, m, \psi_{1}}(x) & =\frac{\psi_{1}(x)-m \psi_{1}(a)}{x-m a} \\
& =\frac{M_{1} x^{2}-f(x)-m f(a)+m M_{1} a^{2}}{x-m a} \\
& =\frac{M_{1}\left(x^{2}-m a^{2}\right)}{x-m a}-\frac{f(x)-m f(a)}{x-m a} .
\end{aligned}
$$

$$
P_{a, m, \psi_{1}}^{\prime}(x)=M_{1} \frac{x^{2}-2 m a x+m a^{2}}{(x-m a)^{2}}-\frac{(x-m a) f^{\prime}(x)-f(x)+m f(a)}{(x-m a)^{2}} .
$$

Since

$$
x^{2}-2 \max +m a^{2}=(x-m a)^{2}+m(1-m) a^{2}>0,
$$

by given condition, we have

$$
M_{1}\left(x^{2}-2 \max +m a^{2}\right) \geq(x-m a) f^{\prime}(x)-f(x)+m f(a) .
$$

This leads to

This implies

$$
\begin{gathered}
M_{1} \frac{x^{2}-2 m a x+m a^{2}}{(x-m a)^{2}} \geq \frac{(x-m a) f^{\prime}(x)-f(x)+m f(a)}{(x-m a)^{2}}, \\
M_{1} \frac{x^{2}-2 m a x+m a^{2}}{(x-m a)^{2}}-\frac{(x-m a) f^{\prime}(x)-f(x)+m f(a)}{(x-m a)^{2}} \geq 0 .
\end{gathered}
$$

$$
P_{a, m, \psi_{1}}^{\prime}(x) \geq 0, \quad \text { for all } x \in[0, m a) \cup(m a, b] .
$$


Similarly, one can show that

$$
P_{a, m, \psi_{2}}^{\prime}(x) \geq 0, \quad \text { for all } x \in[0, m a) \cup(m a, b] .
$$

This gives $P_{a, m, \psi_{1}}$ and $P_{a, m, \psi_{2}}$ are increasing on $x \in[0, m a) \cup(m a, b]$ for all $a \in[0, b]$. Hence by Lemma 2.1, $\psi_{1}(x)$ and $\psi_{2}(x)$ are $m$-convex in $[0, b]$.

Here we give mean value theorems related to functional defined for Petrović's inequality for $m$-convex functions.

Theorem 2.2. Let $\left(x_{1}, \ldots, x_{n}\right) \in[0, b],\left(q_{1}, \ldots, q_{n}\right)$ and $\left(p_{1}, \ldots, p_{n}\right)$ be positive $n$ tuples such that $\sum_{k=1}^{n} p_{k} x_{k} \geq x_{j}$ for each $j=1,2, \ldots, n$. Also, let $\phi(x)=x^{2}$.

If $f \in C^{1}([0, b])$, then there exists $\xi \in(0, b)$ such that

$$
\mathcal{P}_{m}(f)=\frac{(\xi-m a) f^{\prime}(\xi)-f(\xi)+m f(a)}{\xi^{2}-2 m a \xi+m a^{2}} \mathcal{P}_{m}(\phi),
$$

provided that $\mathcal{P}_{m}(\phi)$ is non zero and $a \in(0, b)$.

Proof. As $f \in C^{1}([0, b])$, so there exists real numbers $m_{1}$ and $M_{1}$ such that

$$
m_{1} \leqslant \frac{(x-m a) f^{\prime}(x)-f(x)+m f(a)}{x^{2}-2 \max +m a^{2}} \leqslant M_{1},
$$

for each $x \in[0, b], a \in(0, b)$ and $m \in(0,1)$.

Now let us consider the functions $\psi_{1}$ and $\psi_{2}$ defined in Lemma 2.1. As $\psi_{1}$ is $m$-convex in $[0, b]$,

$$
\mathcal{P}_{m}\left(\psi_{1}\right) \geq 0
$$

that is

$$
\mathcal{P}_{m}\left(M_{1} x^{2}-f(x)\right) \geq 0
$$

which gives

$$
M_{1} \mathcal{P}_{m}(\phi) \geq \mathcal{P}_{m}(f)
$$

Similarly $\psi_{2}$ is $m$-convex in $[0, b]$, therefore one has

$$
m_{1} \mathcal{P}_{m}(\phi) \leqslant \mathcal{P}_{m}(f)
$$

By assumption $\mathcal{P}_{m}(\phi)$ is non zero, combining inequalities (2.10) and (2.11), one has

$$
m_{1} \leqslant \frac{\mathcal{P}_{m}(f)}{\mathcal{P}_{m}(\phi)} \leqslant M_{1} .
$$

Hence, there exists $\xi \in(0, b)$ such that

$$
\frac{\mathcal{P}_{m}(f)}{\mathcal{P}_{m}(\phi)}=\frac{(\xi-m a) f^{\prime}(\xi)-f(\xi)+m f(a)}{\xi^{2}-2 m a \xi+m a^{2}} .
$$

Hence, we get the required result. 
Corollary 2.2. Let $\left(x_{1}, \ldots, x_{n}\right) \in[0, b],\left(q_{1}, \ldots, q_{n}\right)$ and $\left(p_{1}, \ldots, p_{n}\right)$ be positive $n$ tuples such that $\sum_{k=1}^{n} p_{k} x_{k} \geq x_{j}$ for each $j=1,2, \ldots, n$. Also let $\phi(x)=x^{2}$.

If $f \in C^{1}([0, b])$, then there exists $\xi \in(0, b)$ such that

$$
\mathcal{P}(f)=\frac{(\xi-a) f^{\prime}(\xi)-f(\xi)+f(a)}{(\xi-a)^{2}} \mathcal{P}(\phi),
$$

provided that $\mathcal{P}(\phi)$ is non zero and $a \in(0, b)$.

Proof. If we put $m=1$ in (2.9), we get the required result.

Corollary 2.3. Let $\left(x_{1}, \ldots, x_{n}\right) \in[0, b],\left(q_{1}, \ldots, q_{n}\right)$ and $\left(p_{1}, \ldots, p_{n}\right)$ be positive $n$ tuples such that $\sum_{k=1}^{n} p_{k} x_{k} \geq x_{j}$ for each $j=1,2, \ldots, n$ and $a \in(0, b)$. Also let $\phi(x)=x^{2}$.

If $f \in C^{1}([0, b])$, then there exists $\xi \in(0, b)$ such that

$$
\mathcal{P}(f)=f^{\prime \prime}(a) \mathcal{P}(\phi)
$$

Proof. If we put $m=1$ in (2.9), we get

$$
\begin{aligned}
\frac{\mathcal{P}(f)}{\mathcal{P}(\phi)} & =\frac{(\xi-a) f^{\prime}(\xi)-f(\xi)+f(a)}{(\xi-a)^{2}} \\
& =\frac{1}{\xi-a}\left(f^{\prime}(\xi)-\frac{f(a)-f(\xi)}{a-\xi}\right) .
\end{aligned}
$$

Take limit as $\xi \rightarrow a$, we get

$$
\begin{aligned}
\frac{\mathcal{P}(f)}{\mathcal{P}(\phi)} & =\lim _{\xi \rightarrow a} \frac{1}{\xi-a}\left(f^{\prime}(\xi)-\frac{f(a)-f(\xi)}{a-\xi}\right) \\
& =\lim _{\xi \rightarrow a} \frac{1}{\xi-a}\left(f^{\prime}(\xi)-f^{\prime}(a)\right) .
\end{aligned}
$$

Again taking limit as $\xi \rightarrow a$, we get

$$
\frac{\mathcal{P}(f)}{\mathcal{P}(\phi)}=f^{\prime \prime}(a)
$$

Hence, we get the required result.

Theorem 2.3. Let $\left(x_{1}, \ldots, x_{n}\right) \in[0, b],\left(q_{1}, \ldots, q_{n}\right)$ and $\left(p_{1}, \ldots, p_{n}\right)$ be positive $n$ tuples such that $\sum_{k=1}^{n} p_{k} x_{k} \geq x_{j}$ for each $j=1,2, \ldots, n$. Also, let $\phi(x)=x^{2}$.

If $f_{1}, f_{2} \in C^{1}([0, b])$, then there exists $\xi \in(0, b)$ such that

$$
\frac{\mathcal{P}_{m}\left(f_{1}\right)}{\mathcal{P}_{m}\left(f_{2}\right)}=\frac{(\xi-m a) f_{1}^{\prime}(\xi)-f_{1}(\xi)+m f_{1}(a)}{(\xi-m a) f_{2}^{\prime}(\xi)-f_{2}(\xi)+m f_{2}(a)}
$$

provided that the denominators are non-zero and $a \in(0, b)$.

Proof. Suppose a function $k \in C^{1}([0, b])$ be defined as

$$
k=c_{1} f_{1}-c_{2} f_{2},
$$


where $c_{1}$ and $c_{2}$ are defined as

$$
\begin{aligned}
& c_{1}=\mathcal{P}_{m}\left(f_{2}\right), \\
& c_{2}=\mathcal{P}_{m}\left(f_{1}\right) .
\end{aligned}
$$

Then using Theorem 2.2 with $f=k$, one has

$$
(\xi-m a)\left(\left(c_{1} f_{1}-c_{2} f_{2}\right)(\xi)\right)^{\prime}-\left(c_{1} f_{1}-c_{2} f_{2}\right)(\xi)+m\left(c_{1} f_{1}-c_{2} f_{2}\right)(a)=0,
$$

that is

$$
(\xi-m a)\left(c_{1} f_{1}^{\prime}(\xi)-c_{2} f_{2}^{\prime}(\xi)\right)-c_{1} f_{1}(\xi)+c_{2} f_{2}(\xi)+m c_{1} f_{1}(a)-m c_{2} f_{2}(a)=0,
$$

which gives

$$
(\xi-m a) c_{1} f_{1}^{\prime}(\xi)-(\xi-m a) c_{2} f_{2}^{\prime}(\xi)-c_{1} f_{1}(\xi)+c_{2} f_{2}(\xi)+m c_{1} f_{1}(a)-m c_{2} f_{2}(a)=0,
$$

which implies

$$
\begin{aligned}
& c_{1}\left\{(\xi-m a) f_{1}^{\prime}(\xi)-f_{1}(\xi)+m f_{1}(a)\right\}-c_{2}\left\{(\xi-m a) f_{2}^{\prime}(\xi)+f_{2}(\xi)-m f_{2}(a)\right\}=0, \\
& c_{1}\left\{(\xi-m a) f_{1}^{\prime}(\xi)-f_{1}(\xi)+m f_{1}(a)\right\}=c_{2}\left\{(\xi-m a) f_{2}^{\prime}(\xi)-f_{2}(\xi)+m f_{2}(a)\right\}
\end{aligned}
$$

and

$$
\frac{c_{2}}{c_{1}}=\frac{(\xi-m a) f_{1}^{\prime}(\xi)-f_{1}(\xi)+m f_{1}(a)}{(\xi-m a) f_{2}^{\prime}(\xi)-f_{2}(\xi)+m f_{2}(a)} .
$$

After putting the values of $c_{1}$ and $c_{2}$, we get the required result.

Here we state an important lemma that is very helpful in proving mean value theorems related to the non-negative functional of Petrović's inequality for coordinated $m$-convex functions.

Lemma 2.2. Let $\Delta=[0, b] \times[0, d], m \in(0,1)$. Also let $f: \Delta \rightarrow \mathbb{R}$ be a function such that

$$
m_{1} \leqslant \frac{(x-m a) \frac{\partial}{\partial x} f(x, y)-f(x, y)+m f(a, y)}{\left(x^{2}-2 m a x+m a^{2}\right) y^{2}} \leqslant M_{1}
$$

and

$$
m_{2} \leqslant \frac{(y-m c) \frac{\partial}{\partial y} f(x, y)-f(x, y)+m f(x, c)}{\left(y^{2}-2 m c y+m c^{2}\right) x^{2}} \leqslant M_{2},
$$

for all $x \in[0, b] \backslash\{m a\}, a \in(0, b)$ and $y \in[0, d] \backslash\{m c\}, c \in(0, d)$.

Consider the functions $\alpha_{y}:[0, b] \rightarrow \mathbb{R}$, and $\alpha_{x}:[0, d] \rightarrow \mathbb{R}$, defined as

$$
\alpha(x, y)=\max \left\{M_{1}, M_{2}\right\} x^{2} y^{2}-f(x, y)
$$

and

$$
\beta(x, y)=f(x, y)-\min \left\{m_{1}, m_{2}\right\} x^{2} y^{2} .
$$

Then $\alpha$ and $\beta$ are coordinated $m$-convex in $\Delta$. 
Proof. Consider the partial mappings $\alpha_{y}:[0, b] \rightarrow \mathbb{R}$ and $\alpha_{x}:[0, d] \rightarrow \mathbb{R}$ defined by $\alpha_{y}(x):=\alpha(x, y)$ for all $x \in(0, b]$ and $\alpha_{x}(y):=\alpha(x, y)$ for all $y \in(0, d]$.

$$
\begin{aligned}
P_{a, m, \alpha_{y}}(x) & =\frac{\alpha_{y}(x)-m \alpha_{y}(a)}{x-m a} \\
& =\frac{\alpha(x, y)-m \alpha(a, y)}{x-m a} \\
& =\frac{M_{1} x^{2} y^{2}-f(x, y)-m M_{1} a^{2} y^{2}+m f(a, y)}{x-m a} \\
& =M_{1} \frac{\left(x^{2}-m a^{2}\right) y^{2}}{x-m a}-\frac{f(x, y)-m f(a, y)}{x-m a} .
\end{aligned}
$$

So we have

$$
\begin{aligned}
P_{a, m, \alpha_{y}}^{\prime}(x) & =M_{1} \frac{\partial}{\partial x}\left(\frac{\left(x^{2}-m a^{2}\right) y^{2}}{x-m a}\right)-\frac{\partial}{\partial x}\left(\frac{f(x, y)-m f(a, y)}{x-m a}\right) \\
& =M_{1} y^{2} \frac{\left(x^{2}-2 m a x+m a^{2}\right)}{(x-m a)^{2}}-\frac{(x-m a) \frac{\partial}{\partial x} f(x, y)-f(x, y)+m f(a, y)}{(x-m a)^{2}} .
\end{aligned}
$$

Since

$$
M_{1} \geq \frac{(x-m a) \frac{\partial}{\partial x} f(x, y)-f(x, y)+m f(a, y)}{\left(x^{2}-2 m a x+m a^{2}\right) y^{2}},
$$

by given conditions, we have

$$
\left(x^{2}-2 \max +m a^{2}\right) y^{2}>0 .
$$

This implies

$$
\begin{aligned}
& M_{1} y^{2} \frac{\left(x^{2}-2 m a x+m a^{2}\right)}{(x-m a)^{2}} \geq \frac{(x-m a) \frac{\partial}{\partial x} f(x, y)-f(x, y)+m f(a, y)}{(x-m a)^{2}} \\
& M_{1} y^{2} \frac{\left(x^{2}-2 m a x+m a^{2}\right)}{(x-m a)^{2}}-\frac{(x-m a) \frac{\partial}{\partial x} f(x, y)-f(x, y)+m f(a, y)}{(x-m a)^{2}} \geq 0 .
\end{aligned}
$$

This implies

$$
P_{a, m, \alpha_{y}}^{\prime}(x) \geq 0 \text { for all } x \in[0, m a) \cup(m a, b] .
$$

Similarly, one can show that

$$
P_{a, m, \alpha_{x}}^{\prime}(y) \geq 0 \text { for all } x \in[0, m c) \cup(m c, d] .
$$

This ensures that $P_{a, m, \alpha_{y}}$ is increasing on $[0, m a) \cup(m a, b]$ for all $a \in[0, b]$ and $P_{a, m, \alpha_{x}}$ is increasing on $[0, m c) \cup(m c, d]$ for all $c \in[0, d]$. Hence, by Lemma $2.1, \alpha$ is $m$-convex in $\Delta$.

Similarly, one can show that $\beta$ is $m$-convex in $\Delta$.

Here we give mean value theorems related to the functional defined by Petrović's inequality for coordinated $m$-convex functions. 
Theorem 2.4. Let $\Delta=[0, b] \times[0, d],\left(x_{1}, \ldots, x_{n}\right) \in[0, b],\left(y_{1}, \ldots, y_{n}\right) \in[0, d]$ be non-negative $n$-tuples and $\left(q_{1}, \ldots, q_{n}\right),\left(p_{1}, \ldots, p_{n}\right)$ be positive $n$-tuples such that $\sum_{k=1}^{n} p_{k} x_{k} \geq x_{j}$ for each $j=1,2, \ldots, n$. Also, let $\varphi(x, y)=x^{2} y^{2}$.

If $f \in C^{1}(\Delta)$, then there exists $\left(\xi_{1}, \eta_{1}\right)$ and $\left(\xi_{2}, \eta_{2}\right)$ in the interior of $\Delta$, such that

$$
\Upsilon_{m}(f)=\frac{\left(\xi_{1}-m a\right) \frac{\partial}{\partial x} f\left(\xi_{1}, \eta_{1}\right)-f\left(\xi_{1}, \eta_{1}\right)+m f\left(a, \eta_{1}\right)}{\left(\xi_{1}^{2}-2 m a \xi_{1}+m a^{2}\right) \eta_{1}^{2}} \Upsilon_{m}(\varphi)
$$

and

$$
\Upsilon_{m}(f)=\frac{\left(\xi_{2}-m a\right) \frac{\partial}{\partial y} f\left(\xi_{2}, \eta_{2}\right)-f\left(\xi_{2}, \eta_{2}\right)+m f\left(a, \eta_{2}\right)}{\left(\xi_{2}^{2}-2 m a \xi_{2}+m a^{2}\right) \eta_{2}^{2}} \Upsilon_{m}(\varphi)
$$

and provided that $\Upsilon_{m}(\varphi)$ is non-zero and $a \in(0, b)$.

Proof. As $f$ has continuous first order partial derivative in $\Delta$, so there exists real numbers $m_{1}, m_{2}, M_{1}$ and $M_{2}$ such that

$$
m_{1} \leqslant \frac{(x-m a) \frac{\partial}{\partial x} f(x, y)-f(x, y)+m f(a, y)}{\left(x^{2}-2 m a x+m a^{2}\right) y^{2}} \leqslant M_{1}
$$

and

$$
m_{2} \leq \frac{(y-m a) \frac{\partial}{\partial y} f(x, y)-f(x, y)+m f(x, a)}{\left(y^{2}-2 m a y+m a^{2}\right) x^{2}} \leq M_{2},
$$

for all $x \in(0, b], y \in(0, d], a \in(0, b)$ and $m \in(0,1)$.

Now let us consider the functions $\alpha$ and $\beta$ defined in Lemma 2.2.

As $\alpha$ is $m$-convex in $\Delta$, then

$$
\Upsilon_{m}(\alpha) \geq 0
$$

that is

$$
\Upsilon_{m}\left(M_{1} x^{2} y^{2}-f(x, y)\right) \geq 0
$$

which gives

$$
M_{1} \Upsilon_{m}(\varphi) \geq \Upsilon_{m}(f)
$$

Similarly $\beta$ is $m$-convex in $\Delta$, therefore one has

$$
m_{1} \Upsilon_{m}(\varphi) \leqslant \Upsilon_{m}(f)
$$

By the assumption $\Upsilon_{m}(\varphi)$ is non-zero. Combining inequalities (2.14) and (2.15), one has

$$
m_{1} \leqslant \frac{\Upsilon_{m}(f)}{\Upsilon_{m}(\varphi)} \leqslant M_{1}
$$

Hence there exists $\left(\xi_{1}, \eta_{1}\right)$ in the interior of $\Delta$, such that

$$
\Upsilon_{m}(f)=\frac{\left(\xi_{1}-m a\right) \frac{\partial}{\partial x} f\left(\xi_{1}, \eta_{1}\right)-f\left(\xi_{1}, \eta_{1}\right)+m f\left(a, \eta_{1}\right)}{\left(\xi_{1}^{2}-2 m a \xi_{1}+m a^{2}\right) \eta_{1}^{2}} \Upsilon_{m}(\varphi)
$$

Similarly, one can show that

$$
\Upsilon_{m}(f)=\frac{\left(\xi_{2}-m a\right) \frac{\partial}{\partial y} f\left(\xi_{2}, \eta_{2}\right)-f\left(\xi_{2}, \eta_{2}\right)+m f\left(a, \eta_{2}\right)}{\left(\xi_{2}^{2}-2 m a \xi_{2}+m a^{2}\right) \eta_{2}^{2}} \Upsilon_{m}(\varphi)
$$


which is the required result.

Corollary 2.4. Let $\Delta=[0, b] \times[0, d],\left(x_{1}, \ldots, x_{n}\right) \in[0, b],\left(y_{1}, \ldots, y_{n}\right) \in[0, d]$ be non-negative $n$-tuples and $\left(q_{1}, \ldots, q_{n}\right),\left(p_{1}, \ldots, p_{n}\right)$ be positive $n$-tuples such that $\sum_{k=1}^{n} p_{k} x_{k} \geq x_{j}$ for each $j=1,2, \ldots, n$. Also, let $\varphi(x, y)=x^{2} y^{2}$.

If $f \in C^{1}(\Delta)$, then there exists $\left(\xi_{1}, \eta_{1}\right)$ and $\left(\xi_{2}, \eta_{2}\right)$ in the interior of $\Delta$, such that

$$
\Upsilon(f)=\frac{\left(\xi_{1}-a\right) \frac{\partial}{\partial x} f\left(\xi_{1}, \eta_{1}\right)-f\left(\xi_{1}, \eta_{1}\right)+f\left(a, \eta_{1}\right)}{\left(\xi_{1}-a\right)^{2} \eta_{1}^{2}} \Upsilon(\varphi)
$$

and

$$
\Upsilon(f)=\frac{\left(\xi_{2}-a\right) \frac{\partial}{\partial y} f\left(\xi_{2}, \eta_{2}\right)-f\left(\xi_{2}, \eta_{2}\right)+f\left(a, \eta_{2}\right)}{\left(\xi_{2}-a\right)^{2} \eta_{2}^{2}} \Upsilon(\varphi),
$$

provided that $\Upsilon(\varphi)$ is non-zero and $a \in(0, b)$.

Proof. If we put $\mathrm{m}=1$ in (2.12) and (2.13), we get the required result.

Theorem 2.5. Let $\Delta=[0, b] \times[0, d],\left(x_{1}, \ldots, x_{n}\right) \in[0, b],\left(y_{1}, \ldots, y_{n}\right) \in[0, d]$ be non-negative $n$-tuples and $\left(q_{1}, \ldots, q_{n}\right),\left(p_{1}, \ldots, p_{n}\right)$ be positive $n$-tuples such that $\sum_{k=1}^{n} p_{k} x_{k} \geq x_{j}$ for each $j=1,2, \ldots, n$. Also, let $\varphi(x, y)=x^{2} y^{2}$.

If $f_{1}, f_{2} \in C^{1}(\Delta)$, then there exists $\left(\xi_{1}, \eta_{1}\right)$ and $\left(\xi_{2}, \eta_{2}\right)$ in the interior of $\Delta$, such that

$$
\frac{\Upsilon_{m}\left(f_{1}\right)}{\Upsilon_{m}\left(f_{2}\right)}=\frac{\left(\xi_{1}-m a\right) \frac{\partial}{\partial x} f_{1}\left(\xi_{1}, \eta_{1}\right)-f_{1}\left(\xi_{1}, \eta_{1}\right)+m f_{1}\left(a, \eta_{1}\right)}{\left(\xi_{2}-m a\right) \frac{\partial}{\partial x} f_{2}\left(\xi_{2}, \eta_{2}\right)-f_{2}\left(\xi_{2}, \eta_{2}\right)+m f_{2}\left(a, \eta_{2}\right)}
$$

and

$$
\frac{\Upsilon_{m}\left(f_{1}\right)}{\Upsilon_{m}\left(f_{2}\right)}=\frac{\left(\xi_{1}-m a\right) \frac{\partial}{\partial y} f_{1}\left(\xi_{1}, \eta_{1}\right)-f_{1}\left(\xi_{1}, \eta_{1}\right)+m f_{1}\left(a, \eta_{1}\right)}{\left(\xi_{2}-m a\right) \frac{\partial}{\partial y} f_{2}\left(\xi_{2}, \eta_{2}\right)-f_{2}\left(\xi_{2}, \eta_{2}\right)+m f_{2}\left(a, \eta_{2}\right)},
$$

provided that the denominators are non-zero and $a \in(0, b)$.

Proof. Suppose

$$
k=c_{1} f_{1}-c_{2} f_{2},
$$

where $c_{1}$ and $c_{2}$ are defined by

$$
\begin{aligned}
& c_{1}=\Upsilon_{m}\left(f_{2}\right), \\
& c_{2}=\Upsilon_{m}\left(f_{1}\right) .
\end{aligned}
$$


Then using Theorem 2.4 with $f=k$, we get

$$
\begin{aligned}
& (\xi-m a) \frac{\partial}{\partial x}\left(c_{1} f_{1}-c_{2} f_{2}\right)(\xi, \eta)-\left(c_{1} f_{1}-c_{2} f_{2}\right)(\xi, \eta)+m\left(c_{1} f_{1}-c_{2} f_{2}\right)(a, \eta)=0 \\
& (\xi-m a) c_{1} \frac{\partial}{\partial x} f_{1}(\xi, \eta)-(\xi-m a) c_{2} \frac{\partial}{\partial x} f_{2}(\xi, \eta)-c_{1} f_{1}(\xi, \eta)+c_{2} f_{2}(\xi, \eta) \\
& +m c_{1} f_{1}(a, \eta)-m c_{2} f_{2}(a, \eta)=0, \\
& c_{1}\left\{(\xi-m a) \frac{\partial}{\partial x} f_{1}(\xi, \eta)-f_{1}(\xi, \eta)+m f_{1}(a, \eta)\right\}-c_{2}\left\{(\xi-m a) \frac{\partial}{\partial x} f_{2}(\xi, \eta)\right. \\
& \left.+f_{2}(\xi, \eta)-m f_{2}(a, \eta)\right\}=0, \\
& c_{1}\left\{(\xi-m a) \frac{\partial}{\partial x} f_{1}(\xi, \eta)-f_{1}(\xi, \eta)+m f_{1}(a, \eta)\right\}=c_{2}\left\{(\xi-m a) \frac{\partial}{\partial x} f_{2}(\xi, \eta)\right. \\
& \left.-f_{2}(\xi, \eta)+m f_{2}(a, \eta)\right\},
\end{aligned}
$$

and

$$
\frac{c_{2}}{c_{1}}=\frac{\left(\xi_{1}-m a\right) \frac{\partial}{\partial x} f_{1}\left(\xi_{1}, \eta_{1}\right)-f_{1}\left(\xi_{1}, \eta_{1}\right)+m f_{1}\left(a, \eta_{1}\right)}{\left(\xi_{2}-m a\right) \frac{\partial}{\partial x} f_{2}\left(\xi_{2}, \eta_{2}\right)-f_{2}\left(\xi_{2}, \eta_{2}\right)+m f_{2}\left(a, \eta_{2}\right)} .
$$

Similarly, one can show that

$$
\frac{c_{2}}{c_{1}}=\frac{\left(\xi_{1}-m a\right) \frac{\partial}{\partial y} f_{1}\left(\xi_{1}, \eta_{1}\right)-f_{1}\left(\xi_{1}, \eta_{1}\right)+m f_{1}\left(a, \eta_{1}\right)}{\left(\xi_{2}-m a\right) \frac{\partial}{\partial y} f_{2}\left(\xi_{2}, \eta_{2}\right)-f_{2}\left(\xi_{2}, \eta_{2}\right)+m f_{2}\left(a, \eta_{2}\right)} .
$$

After putting the values of $c_{1}$ and $c_{2}$, we get the required result.

\section{Log Convexity}

Here we have defined some families of parametric functions which we use in sequal. Let $I=[0, a), J=[0, b) \subseteq \mathbb{R}$ be intervals and $f_{t}: I \times J \rightarrow \mathbb{R}$ represents some parametric mapping for $t \in(c, d) \subseteq \mathbb{R}$. We define functions

$$
f_{t, y}: I \rightarrow \mathbb{R} \text { by } f_{t, y}(u)=f_{t}(u, y)
$$

and

$$
f_{t, x}: J \rightarrow \mathbb{R} \text { by } f_{t, x}(v)=f_{t}(x, v),
$$

where $x \in I$ and $y \in J$. Suppose $\mathcal{H}_{1}$ denotes the class of functions $f_{t}: I \times J \rightarrow \mathbb{R}$ for $t \in(c, d)$ such that the functions

$$
t \mapsto r_{m}\left(u_{0}, u_{1}, u_{2}, f_{t, y}\right), \quad \text { for all } u_{0}, u_{1}, u_{2} \in I
$$

and

$$
t \mapsto r_{m}\left(v_{0}, v_{1}, v_{2}, f_{t, x}\right), \quad \text { for all } v_{0}, v_{1}, v_{2} \in J
$$

are log-convex functions in Jensen sense on $(c, d)$.

The following lemma is given in [16]. 
Lemma 3.1. Let $I \subseteq \mathbb{R}$ be an interval. A function $f: I \rightarrow(0, \infty)$ is log-convex in $J$-sense on $I$, that is, for each $r, t \in I$

$$
f(r) f(t) \geq f^{2}\left(\frac{t+r}{2}\right)
$$

if and only if the relation

$$
m^{2} f(t)+2 m n f\left(\frac{t+r}{2}\right)+n^{2} f(r) \geq 0
$$

holds, for each $m, n \in \mathbb{R}$ and $r, t \in I$.

Our next result comprises properties of functional defined in Theorem 2.1.

Theorem 3.1. Let $f_{t} \in \mathcal{H}_{1}$ and $\Upsilon_{m}$ be the functional defined in (2.8). Then the function $t \mapsto \Upsilon_{m}\left(f_{t}\right)$ is log-convex in Jensen sense for each $t \in(c, d)$.

Proof. Let

$$
h(u, v)=m^{2} f_{t}(u, v)+2 m n f_{\frac{t+r}{2}}(u, v)+n^{2} f_{r}(u, v),
$$

where $m, n \in \mathbb{R}$ and $t, r \in(c, d)$. Also we can consider that

$$
h_{y}(u)=m^{2} f_{t, y}(u)+2 m n f_{\frac{t+r}{2}, y}(u)+n^{2} f_{r, y}(u)
$$

and

which gives

$$
h_{x}(v)=m^{2} f_{t, x}(v)+2 m n f_{\frac{t+r}{2}, x}(v)+n^{2} f_{r, x}(v),
$$

$$
\begin{aligned}
r_{m}\left(u_{0}, u_{1}, u_{2}, h_{y}\right)= & m^{2} r_{m}\left(u_{0}, u_{1}, u_{2}, f_{t, y}\right)+2 m n r_{m}\left(u_{0}, u_{1}, u_{2}, f_{\frac{t+r}{2}, y}\right) \\
& +n^{2} r_{m}\left(u_{0}, u_{1}, u_{2}, f_{r, y}\right) .
\end{aligned}
$$

As $r_{m}\left[u_{0}, u_{1}, u_{2}, f_{t, y}\right]$ is log-convex in Jensen sense so by using Lemma 3.1 , the right hand side of the above expression is non negative so $h_{y}$ is $m$-convex, similarly $h_{x}$ is also $m$-convex, so $h$ is $m$-convex on coordinates, which implies $r_{m}(h) \geq 0$ and

$$
m^{2} r_{m}\left(f_{t}\right)+2 m n r_{m}\left(f_{\frac{t+r}{2}}\right)+n^{2} r_{m}\left(f_{r}\right) \geq 0 .
$$

Hence, $t \mapsto \Upsilon_{m}\left(f_{t}\right)$ is log-convex in Jensen sense.

Theorem 3.2. Assume that $f_{t}$ is of class $\mathcal{H}_{1}$ and $\Upsilon_{m}$ be the functional defined in (2.8). If the function $\Upsilon_{m}\left(f_{t}\right)$ is continuous for each $t \in(c, d)$, then $\Upsilon_{m}\left(f_{t}\right)$ is log-convex for each $t \in(c, d)$.

Proof. If a function is continuous and log-convex in Jensen sense, then it is log-convex (see [3, p. 48]). It is given that $\Upsilon_{m}\left(f_{t}\right)$ is continuous for each $t \in(c, d)$, hence $\Upsilon_{m}\left(f_{t}\right)$ is log-convex for each $t \in(c, d)$.

Lemma 3.2. If $f$ is a convex function for all $x_{1}, x_{2}, x_{3}$ of an open interval $I$ for which $x_{1}<x_{2}<x_{3}$, then

$$
\left(x_{3}-x_{2}\right) f\left(x_{1}\right)+\left(x_{1}-x_{3}\right) f\left(x_{2}\right)+\left(x_{2}-x_{1}\right) f\left(x_{3}\right) \geq 0 .
$$


Theorem 3.3. Let $f_{t} \in \mathcal{H}_{1}$ and $\Upsilon_{m}$ be the functional defined in (2.8). If $\Upsilon_{m}\left(f_{t}\right)$ is positive, then for some $r<s<t$, where $r, s, t \in(c, d)$, one has

$$
\left[\Upsilon_{m}\left(f_{s}\right)\right]^{t-r} \leq\left[\Upsilon_{m}\left(f_{r}\right)\right]^{t-s}\left[\Upsilon_{m}\left(f_{t}\right)\right]^{s-r}
$$

Proof. Consider the functional $\Upsilon_{m}\left(f_{t}\right)$. Also let $r<s<t$, where $r, s, t \in(c, d)$, since $\Upsilon_{m}\left(f_{t}\right)$ is $\log$-convex, that is, $\log \Upsilon_{m}\left(f_{t}\right)$ is convex. By taking $f=\log \Upsilon_{m}$ in Lemma 3.2 , we have

$$
(t-s) \log \Upsilon_{m}\left(f_{r}\right)+(r-t) \log \Upsilon_{m}\left(f_{s}\right)+(s-r) \log \Upsilon_{m}\left(f_{t}\right) \geq 0,
$$

which can be written as

$$
\left[\Upsilon_{m}\left(f_{s}\right)\right]^{t-r} \leq\left[\Upsilon_{m}\left(f_{r}\right)\right]^{t-s}\left[\Upsilon_{m}\left(f_{t}\right)\right]^{s-r}
$$

Acknowledgements. The authors are very grateful to the editor and reviewers for their careful and meticulous reading of the paper. The research work of the 2 nd author is supported by Higher Education Commission of Pakistan under NRPU 2016, Project No. 5421.

\section{REFERENCES}

[1] M. Alomari and M. Darus, On the Hadamard's inequality for log convex functions on coordinates, J. Inequal. Appl. 2009(1) (2009), 13 pages.

[2] M. K. Bakula, J. Pečarić and M. Ribičić, Companion inequalities to Jensen's inequality for mconvex and $(\alpha, m)$-convex functions, Journal of Inequalities in Pure and Applied Mathematics $7(5)$ (2006), 32 pages.

[3] P. S. Bullen, Handbook of Means and Their Inequalities, Springer Science \& Business Media, Dordrecht, Boston, London, 2013.

[4] S. Butt, J. Pečarić and A. U. Rehman, Exponential convexity of Petrović and related functional, J. Inequal. Appl. 2011(1) (2011), 16 pp.

[5] S. S. Dragomir, On some new inequalities of Hermite-Hadamard type for $m$-convex functions, Tamkang J. Math. 33(1) (2002), 45-56.

[6] S. S Dragomir, On Hadamards inequality for convex functions on the co-ordinates in a rectangle from the plane, Taiwanese J. Math. 5(4) (2001), 775-788.

[7] S. S. Dragomir, Charles E.M. Pearce, Selected Topics on Hermite-Hadamard Inequalities and Applications, RGMIA Monographs, Victoria University, Melbourne, 2000.

[8] G. Farid, M. Marwan and A. U. Rehman, New mean value theorems and generalization of Hadamard inequality via coordinated m-convex functions, J. Inequal. Appl. 2015(1) (2015), 11 pages.

[9] M. Krnić, R. Mikić and J. Pečarić, Strengthened converses of the Jensen and Edmundson-LahRibarič inequalities, Adv. Oper. Theory 1(1) (2016), 104-122.

[10] T. Lara, E. Rosales and J. L. Sánchez, New properties of $m$-convex functions, Int. J. Appl. Math. Anal. Appl. 9(15) (2015), 735-742.

[11] V. G. Mihesan, A generalization of the convexity, Seminar on Functional Equations, Approximation and Convexity, Cluj-Napoca, Romania, 1993.

[12] Z. Pavić and M. A. Ardiç, The most important inequalities of $m$-convex functions, Turkish J. Math. 41(3) (2017), 625-635.

[13] J. E. Pečarić, On the Petrović's inequality for convex functions, Glas. Mat. 18(38) (1983), 77-85. 
[14] J. Pečarić and V. Čuljak, Inequality of Petrović and Giaccardi for convex function of higher order, Southeast Asian Bull. Math. 26(1) (2003), 57-61.

[15] J. E. Pečarić, F. Proschan and Y. L. Tong, Convex Functions, Partial Orderings and Statistical Applications, Academic Press, New York, 1991.

[16] J. Pečarić and A. U. Rehman, On logarithmic convexity for power sums and related results, J. Inequal. Appl. 2008(1) (2008), 10 pages.

[17] M. Petrović, Sur une fonctionnelle, Publ. Inst. Math. (Beograd) (1932), 146-149.

[18] J. Pečarić and J. Peric, Improvements of the Giaccardi and the Petrovic inequality and related Stolarsky type means, An. Univ. Craiova Ser. Mat. Inform. 39(1) (2012), 65-75.

[19] A. U. Rehman, M. Mudessir, H. T. Fazal and G. Farid, Petrović's inequality on coordinates and related results, Cogent Math. 3(1) (2016), 11 pp.

[20] J. Rooin, A. Alikhani and M. S. Moslehian, Operator m-convex functions, Georgian Math. J. 25(1) (2018), 93-107.

[21] G. Toader, On a generalization of the convexity, Mathematica 30(53) (1988), 83-87.

[22] G. Toader, Some generalizations of the convexity, in: I. Marusciac and W. W. Breckner (Eds.), Proceedings of the Colloquium on Approximation and Optimization, University of Cluj-Napoca, 1984.

[23] X. Zhang and W. Jiang, Some properties of log-convex function and applications for the exponential function, Comput. Math. Appl. 63(6) (2012), 1111-1116.

[24] B. Xi, F. Qi and T. Zhang, Some inequalities of Hermite-Hadamard type for m-harmonicarithmetically convex functions, ScienceAsia 41(51) (2015), 357-361.

${ }^{1}$ COMSATS University Islamabad, Attock Campus, ATTOCK, PAKISTAN

Email address: atiq@mathcity.org

Email address: ghlmfarid@cuiatk.edu.pk, faridphdsms@hotmail.com

${ }^{2}$ Government Post Graduate College Attock, AtTock, Pakistan

Email address: waseem.iqbal.attock@gmail.com 